\title{
A Chain Topology for Efficient Monitoring of Food Grain Storage Using Smart Sensors
}

\author{
Alekha Kumar Mishra ${ }^{1}$, Asis Kumar Tripathy ${ }^{1}$, Mohammad S. Obaidat, Fellow of IEEE and Fellow of \\ $S C S^{2}$, Zhiyuan $\operatorname{Tan}^{3}$, Mukesh Prasad ${ }^{4}$, Balqies Sadoun ${ }^{5}$ and Deepak Puthal ${ }^{4}$ \\ ${ }^{1}$ Vellore Institute of Technology, Vellore, India \\ ${ }^{2}$ University of Jordan, Jordan \\ ${ }^{2}$ Nazarbayev University, Astana, Kazakhstan \\ ${ }^{3}$ Edinburgh Napier University, UK \\ ${ }^{4}$ University of Technology Sydney, Australia \\ ${ }^{5}$ Al Balqa' Applied University, Jordan \\ Corresponding Email: \{alekha, msobaidat, deepak.puthal\}@gmail.com
}

Keywords: $\quad$ Food grain storage monitoring, Smart Sensor Devices, Sensor Networks, Network Topology, Chain topology

Abstract: Due to lack of an efficient monitoring system to periodically record environmental parameters for food grain storage, a huge loss of food grains in storage is reported every year in many developing countries, especially south-Asian countries. Although Smart Sensor Networks have been successfully implemented in various applications such as health-care, military, and wildlife monitoring, there are still various issues to be addressed in food grain storage monitoring applications. Due to the food grain storage infrastructure constraints, the commonly practiced network topologies of sensor devices such as mesh, star, and grid cannot provide an effective monitoring environment. In this paper, we proposed a topology using smart sensors that can effectively cover and monitor the food grain storage area. It uses a chained structure of sensor devices with directional antennas to accurately sense and report the environmental data. The proposed topology works better than common topologies due to its chain-based structure which remains unaffected by various hindrance imposed due to food grain storage infrastructure. From the experimental results it is conclude that the proposed topology has effective coverage percentage, detection accuracy, and message delivery over Cluster-based and Mesh topologies in food grain storage environments.

\section{Introduction}

Due to climatic changes, irregularities were observed in weather conditions in recent years. These changes directly affect the longevity and utility period of food grains. It is reported that the wheat and rice production is passing the record level every year in south-Asian countries. However, due to lack of adequate monitoring infrastructure and process, the loss of food grains in these years apprehend the productivity (Manay and M.Shadaksharaswamy, 2008; Sawant et al., 2012). Irregular changes in weather conditions provides favorable conditions for growth of insect, pests, molds, rodents, fungi, and mycotoxins in food grain storage. It is reported that around twenty percent of food grains are wasted in food storage. The above fact demands a monitoring and control environment for food grain in food depot across the agricultural zones of a country. In this environment, the influential factors of the food storage depots are continuously observed to maintain favorable scenario for food grains. It is found that traditional or manual food grain storage monitoring by store officials is inefficient as they cannot reach to all locations on regular basis. The smart sensor devices are playing these years in the sensing domain these days where these devices detect and collect relevant environmental data such as temperature, atmospheric pressure, humidity, and light (Sahoo et al., 2012; Sharma et al., 2017b). Securing such infrastructure is also playing a vital role in current communication infrastructure (Puthal et al., 2018). Therefore, a smart sensor network can be effectively used for monitoring influencing parameters in food grain storage (Hadjidj et al., 2013; Carlos-Mancilla et al., 2016). It is observed from the literature that the smart sensor devices are 
commonly deployed in free space. If more number of smart sensors are involved for a particular monitoring application, then it is found that there exists sufficient free space for direct communication between these devices. These common deployment structure is provided by various network topologies such as tree, mesh, grid, star, and clustered. However, these topologies may not be suitable for food grain storage monitoring application, since the food grain storage are often filled with grain bins and there is hardly a clear line of sight inside the storage area of bins. This paper devises an effective topology of smart sensors to monitor influential environmental parameters in a food grain storage depot. It is efficient and more suitable for this monitoring environment compared to mesh, tree, and clustered topology. It is shown that the proposed topology can perform effectively with sensor devices equipped with directional antenna and sectored sensing disc. The performance of the proposed topology is compared with mesh and clustered topology using simulation experiments and found to be better in terms of detection accuracy, energy efficiency, and message delivery ratio. The sections of this contribution are organized in the following way. Section 2 provides a detailed report on infrastructure of food grain storage depot. Section 3 summaries the characteristics along with pros and cons of existing smart sensor network topologies. Section 4 provides an analysis on unsuitability of existing topologies for monitoring food grain storage depot. Section 5 elaborates on the structure and operational details of the proposed topology for food grain storage monitoring. Section 6 analyzes the coverage and energy efficiency of the proposed topology. Section 7 summarizes simulation results of comparison between proposed topology and existing competing schemes followed by concluding remarks in Section 8 .

\section{SURVEY OF FOOD GRAIN STORAGE INFRASTRUCTURE}

Almost a common standard is followed throughout the globe for constructing infrastructure for food grain storage depot. The dimension and facilities may vary up to some extent depending on the kind of food grain. The most common shape of storage is pyramid dome shaped as shown in Figure 1. The standard storage area of the depot is 126 meters $\times 22$ meters. The height of the depot varies from 5.5 meters to 6.25 meters. The entire capacity of the depot is divided into 3 compartments with each compartment of capacity 5000 metric ton (MT). The number of stacks for each compartment is twelve. The ventilators are

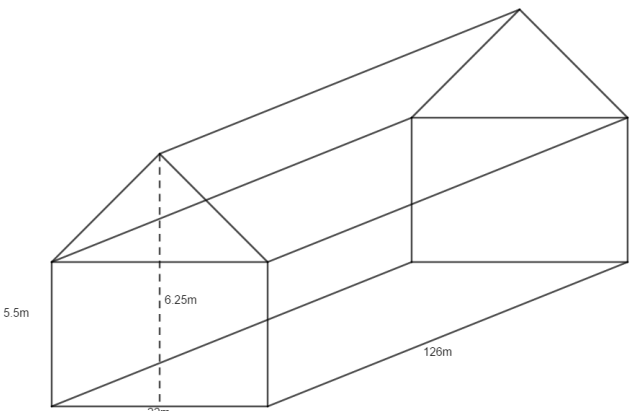

Figure 1: The dimension and shape of a food grain depot.

used on both top and bottom side of the walls. The stack size is approximately 6 meters $\times 10$ meters. The Galvanized Iron Corrugated (GIC) silos storage are used for longterm storage in selected depots where food grain may be stored for a longer period (years). GIC silos are quite expensive compared to standard storage depot (Deshpande et al., 2010). Additionally, cover and plinth (CAP) storage structures are the most common among the farmers. Sometimes, cover and plinth structures are also practiced by food grain depot officials to store excess amount of food grains with depot's premise. Sufficient space are provided between food grain stacks inside a depot to facilitate smooth movement of carriage vehicles. But, during post-harvesting months the food grain depots are often full occupied with food grain bins.

The essential parameters that are monitored regularly in the food grain depot is temperature and air flow inside depot, and moisture content of the food grains. Table 1 shows the drying temperature and maximum moisture level maintained for different types of food grains (Hellevang, 2010).

Table 1: Moisture content levels and drying temperature for various food grains.

\begin{tabular}{|l|c|c|}
\hline Food grain & $\begin{array}{c}\text { Drying } \\
\text { Temperature }\left({ }^{\circ} \mathbf{C}\right)\end{array}$ & $\begin{array}{c}\text { Max. Moisture } \\
\text { Content }(\boldsymbol{\%})\end{array}$ \\
\hline Paddy & 60 & 17 \\
\hline Wheat & 65 & 14.5 \\
\hline Oats & 60 & 14 \\
\hline Barley & 55 & 14.8 \\
\hline Maize & 49 & 14 \\
\hline Flax & 80 & 10 \\
\hline Peas & 45 & 16 \\
\hline
\end{tabular}

It is reported that these parameters are continuously monitored and when these parameters reaches a level beyond the mentioned range due to changes in environment, it requires the manual attention of the food grain store officials for further action. Smart sensors are a suitable options to replace the manual monitor- 
ing process. A set of smart sensor devices may be deployed to form a smart network to facilitate the monitoring process. In the following section, a survey of existing smart network topology is provided for food grain storage monitoring.

\section{SURVEY OF SMART SENSOR NETWORK TOPOLOGY}

It has always been a challenging task to plan a suitable network topology for a target application. It is required that the designed network topology must satisfy the desired coverage, connectivity, coverage, and network lifetime (Fan and Jin, 2010). The topologies as reported in the literature are mostly designed based on the target application requirements (Ceclio and Furtado, 2014). A brief survey of these topologies is provided below.

Star topology is a single-hop system, where all sensors communicate directly to the gateway. This is simple and efficient for small networks (Yang et al., 2017). Here, a single node failure is not an issue since it does not affect the communication process of other nodes. There can be more network segments branching out from the central gateway node. The energy consumption for communication is limited to the distance between a node and its gateway. This topology is suitable for network of smaller size limited to geographical area of approximately 100 meters. When it is required to be deployed in large scale, the star topology is applied with hierarchical schemes. In a tree topology, the sink node also known as base station (BS) is considered as the root of the network tree. The root node is connected with relay nodes; those are responsible for forwarding sensed data to the root. The relaying nodes forms the network with multiple levels with root as sink node (Sharma et al., 2017a). Sometime the relay nodes are equipped with sensors in order to perform both sensing and relaying task. The tree topology can be scalable to desired size with a minimum effort. The overall energy consumption of the network is lower because of the small range nodeto-node communication. The data transmission from sensing nodes to the sink node requires the coordination of intermediate relaying nodes. A mesh topology mostly resembles a mathematical graph structure, where each node is in connection with all other nodes located with its communication range. An efficient multi-hop communication scheme is essential for this topology. Since, intermediate nodes in the path to sink node play the role of forwarding node, it is necessary to ensure that the path to sink node is connected before sending data. Mesh topology based networks are excellent for fault tolerance due to existence of multiple path to sink nodes. The characteristics of existing multi-hop communication scheme can be easily modified to support larger network. In a Grid topology, the network is divided into a rectangular grid, where sensor devices are deployed at each grid point(Roy et al., 2018). The commonly adapted grid layout are unit square, equilateral triangle, and hexagon. The square grid topology is more popular compared to its counterparts due to having natural placement characteristics. A grid topology is an ideal condition of topology in terms of coverage of network. Since, it is really difficult to achieve optimal performance with basic grid topology, often more than one node are placed on a square grid. Clustered topology is most adaptive and energy-efficient for most common applications. As it is named, the topology consists of a number of clusters, where each cluster comprises a group of nodes located geographically closer to each other. A designated node called cluster-head $(\mathrm{CH})$ is responsible for coordination of nodes within a cluster and forwarding data of the nodes to the sink node. The task of non- $\mathrm{CH}$ nodes is limited to sensing and sending data to $\mathrm{CH}$. There may be bridge to connect $\mathrm{CHs}$ and facilitate inter-cluster communication. The bridge nodes significantly reduce the energy consumption of inter-cluster communications. The clustered topology has the lowest communication overhead among all topologies due to minimum number of message communications per round. Moreover, hierarchical structure simplifies the process of routing messages.

Some applications demand the combination of more than one previously discussed topologies to improve the performance (Aziz et al., 2013). The hybrid approaches are scalable up to thousands of nodes without deteriorating the network operations. Clusteredtree topology forms tree among the clusters in the network (Hong et al., 2016). It uses multilevel heterogeneous features to define network structure. It takes the hierarchical structure advantage from tree, and utilizes optimal network communication scheme of clusters. Cluster-star topology is a hybrid of star and clustered topology. It uses the simplified structure of star topology at the lower end of the network while utilizing cluster based communication at the higher level. Cluster-mesh topology divides the deployment area into number of cells. Here, the term cells is referred to rectangular areas of equal size (Alsemairi and Younis, 2016). Each cell forms a cluster among the nodes in the cell. Thereafter, an inter- $\mathrm{CH}$ mesh network is formed for inter-cluster communication. The sink node participates in the topology as a cluster head and does not appear just as the end point of data 
routes. Table 2 summaries the strengths and limitations of the above discussed topologies.

\section{ANALYSIS OF EXISTING TOPOLOGIES FOR FOOD GRAIN STORAGE MONITORING}

The first and foremost requirement of a food grain storage structure is that network deployment must not utilize the open space available within the depot. It is reported that placing sensing devices in this space would hinder the process of bulk loading and unloading of food grains. Secondly, the sensing devices cannot be deployed near the food grain bins as the bins stacks are displaced frequently from one place to another depending upon the space requirements. In this case, devices deployment locations are required to change every time; the bins are relocated or displaced. Moreover, sensing devices placed in these locations may be prone to physical damage. Finally, there is no additional support such as pillars near the food grain bins to fix sensing devices permanently. Based on the above requirements and constraints it is found that the only option available to deploy the network is to utilize the inner walls and ceiling of the depot. The topology like star, tree, mesh, grid, and clusters cannot be used efficiently in food grain depot. This is because, all the above mentioned topologies and the hybrids requires the deployment of the nodes in the region of interest. Additionally, these topologies need open space between nodes for communication in all direction because of the omni-directional antenna equipped with these devices. The star topology is feasible up to some extent; however, it is difficult to find a suitable location for gateway node to stay in the communication range of all the devices. All clustered topologies require the sensing devices to be deployed near the food grains, therefore are not suitable for food grain storage. The grid topology is suitable for food grain depot if the grid is deployed on the inner walls. However, the sensing range may be limited due to omni-directional antenna. Moreover it requires significantly more number of sensor devices. The tree based topology may also require significantly large number of nodes when deployed on the inner walls of the depot. Table 3 summarizes the unsuitability of existing topologies for food grain storage monitoring. It is observed from the above discussion that the sensing devices with directional antenna and sectored sensing disc are more suitable for indoor applications compared to their counterparts (Yu et al.,
2011).

\section{PROPOSED WORK}

In this section, the proposed topology of smart sensor devices is presented for efficiently monitoring food grain storages. This topology is a double ended chain structure and it is named as two-tail chain topology. It consists of smart sensors that form a chain structure with the following additional features. The node deployed on the top of the chain is the head node also known as the gateway node. It is responsible for collecting data from all nodes and forwarding it to the relaying node of the network. An additional relaying node is connected to the gateway node. It is responsible for forwarding the data collected from gateway node to the sink node. It is assumed that the relaying node can communicate to sink node either directly or via multi-hop communication. The sensing devices are equipped with directional communication antenna and sensors with sectored sensing disc. Each node in the network can sense and communicate data except gateway and relaying node. The process of sending sensed data begins from both ends (also known as tails) of the chain. It is then forwarded to the next node toward the location of gateway in the chain until it reaches gateway node. In the following subsections, the structure and functions of proposed topology is discussed in details.

\subsection{Structural Details of Proposed Topology}

Figure 2 shows the deployment structure of the proposed topology. Two nodes at the end of the chain are called tail nodes. The tail nodes and the gateway nodes are placed at the opposite ends across the length of the storage depot. The inner walls across the length of the storage depot are used to deploy the chain of sensing devices. The simplest structure of the chain consists of only one chain on each of the walls. More than one chain can be deployed on each wall and ceiling connecting to gateway depending on the coverage requirements. The above topology of sensing devices is effective only if the following type of device positioning structure is followed. Figure 3 shows the direction of communicating radio antenna of sensing devices in the chain. Since the major task of these devices is to sense temperature and moisture data and send them to gateway node, the communicating sector range of the directional antenna are positioned towards the upper level (gateway end) of the chain. Figure 4 shows the sensing rage of the devices. Since devices with sectored sensors have long range sens- 
Table 2: Summary of pros and cons of smart sensor network topologies.

\begin{tabular}{|l|l|l|}
\hline Topology & Strengths & Limitations \\
\hline Star & $\begin{array}{l}\text { Overall lower power consumption } \\
\text { Small area network (100 meters) }\end{array}$ & Limited communication \\
\hline Tree & $\begin{array}{l}\text { Lower power consumption } \\
\text { Scalable }\end{array}$ & $\begin{array}{l}\text { Nodes are time synchronized } \\
\text { Requires coordination among the nodes }\end{array}$ \\
\hline Mesh & $\begin{array}{l}\text { Fault tolerant } \\
\text { Scalable }\end{array}$ & $\begin{array}{l}\text { Redundancy } \\
\text { Higher energy consumption }\end{array}$ \\
\hline Grid & Ideal condition for coverage & Not practical \\
\hline Cluster & $\begin{array}{l}\text { Minimized no. of messages } \\
\text { Simplified routing }\end{array}$ & Cluster-head selection overhead \\
\hline Cluster-Tree & $\begin{array}{l}\text { Lower power consumption } \\
\text { Improved network lifetime }\end{array}$ & $\begin{array}{l}\text { Computational overheads } \\
\text { Cluster-Star }\end{array}$ \\
$\begin{array}{l}\text { Lower energy } \\
\text { Scalable }\end{array}$ & Additional hardwares \\
\hline Cluster-Mesh & Lower energy & Higher node density \\
\hline
\end{tabular}

Table 3: Unsuitability of smart sensor network topologies

\begin{tabular}{|l|l|}
\hline Topology & The reason for unsuitability \\
\hline Star & $\begin{array}{l}\text { Difficulty in positioning the gateway } \\
\text { node }\end{array}$ \\
\hline Tree & Requires open space \\
\hline Mesh & $\begin{array}{l}\text { Requires significantly higher num- } \\
\text { ber of nodes }\end{array}$ \\
\hline Grid & Limited sensing range \\
\hline Cluster & $\begin{array}{l}\text { Requires open space for inter- } \\
\text { cluster communication }\end{array}$ \\
\hline
\end{tabular}

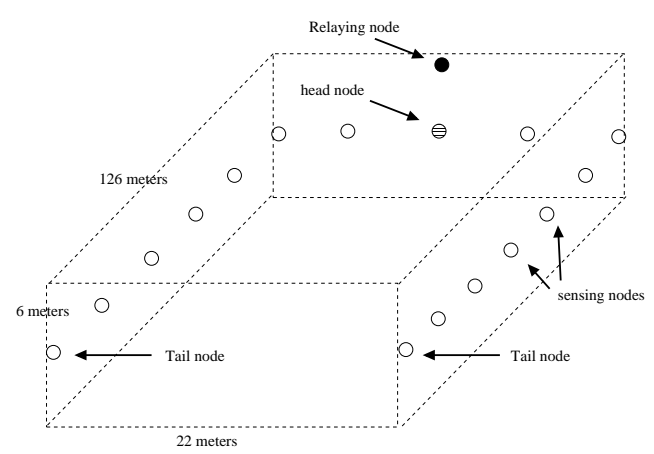

Figure 2: The position of sensing devices in the chained structure.

ing ability compared to omni-directional sensors, the sensing discs are positioned facing the opposite wall of the depot to achieve accurate sensing from the mid area of the depot.

\subsection{Energy Efficient Data Aggregation}

Here, it is assumed that the sensing devices are time synchronized for data collection and aggregation process. The data collection begins at tail nodes. In each

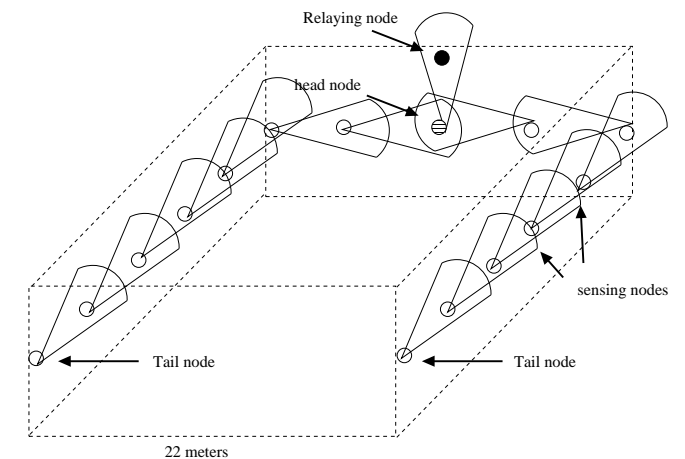

Figure 3: Communication range of sensors with directional antenna.

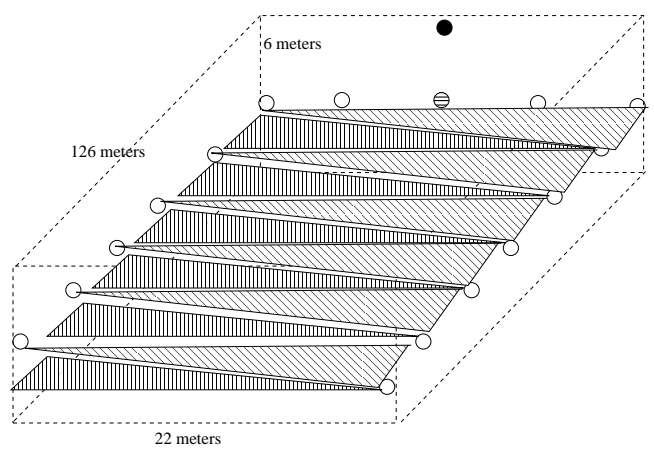

Figure 4: Sensing range of the sensors.

round of data collection, tail nodes send data to the next node towards gateway in the chain. The intermediate nodes in the chain aggregate the received data from tail end with its own data and forward them to the next node towards gateway. Algorithm 1 shows this process of aggregation. The node in the communication range of gateway can directly send the data to gateway. 


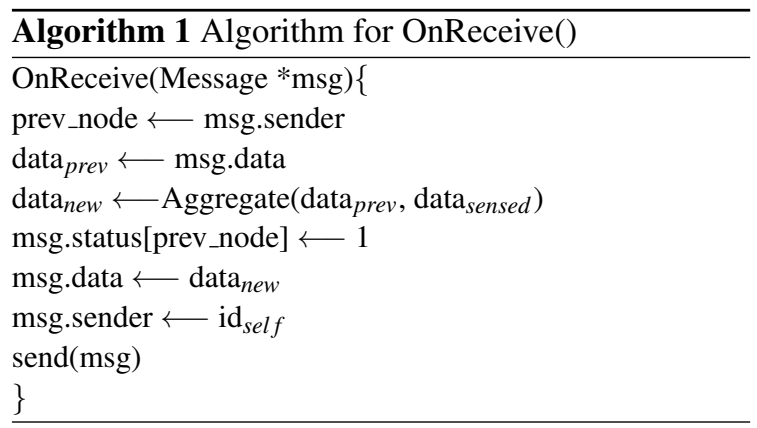

\subsection{Communication between relaying node and sink node}

Upon receiving the aggregated data from both ends of the chain, gateway node sends it to the relaying node. The number of relaying nodes in the network depends on the number of food grain depots under the processing of monitoring. The following existing topologies can be adapted depending on the location of sink and number of relaying nodes:

- Star topology: If it is possible to locate the sink node around the mid point of the area of the food grain depot center, then star topology would be more suitable for this part of the communication. In this case, all relaying nodes can directly communicate with the sink node. Thus, the process of forwarding aggregated data by a relaying node is independent of other relaying nodes in the network.

- Tree topology: When all relaying nodes are not in the communication range of the sink node and number of relaying nodes is large, then it is appropriate to use tree topology of relaying nodes with sink node as root. A simple multi-level routing scheme (Zin et al., 2014; Tanwar et al., 2015) can be used to forward the aggregated data to the sink node using this topology.

\subsection{Communication under node failure}

To synchronize the process of the data aggregation, each node waits for a specific period of time before initiating its data transfer. When the data arrives from the tail end before the timer expires, then the node follows the normal aggregation and forwards process as discussed before. However, if the data does not arrives before the timer expires, then node initiates a fresh data transfer along with a failure status bit for the previous node in the chain. Algorithm 2 represents this communication process. Failure of a node leads to a break of a link in the chain; only if

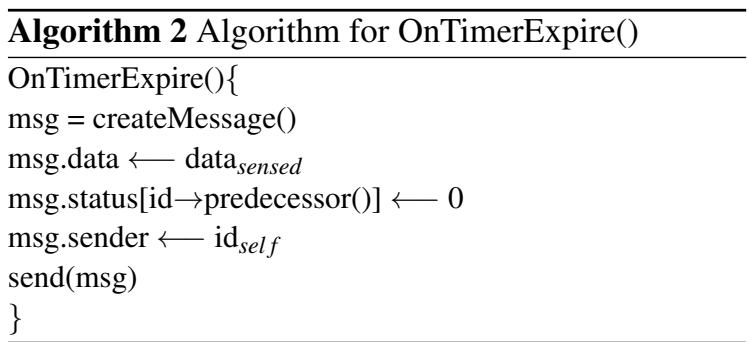

each node has exactly one node in its communication range. The tolerance of connectivity can be improved by increasing the node coverage across the chain to greater than or equal to 2 . This implies that the connectivity failure tolerance is directly proportional to the node density across the chain.

\section{ANALYSIS}

\subsection{Network size and coverage}

The proposed two-tail chain topology can collect data efficiently with minimum number of nodes compared to existing topologies. This is possible due to the chain structure of sensor devices, and positioning of their directional sensing disc. Since devices also uses directional antenna for communication and positioned across the line of the chain, the proposed topology is able to provide a clear space for communication. Whereas, the topologies such as mesh, tree, and cluster-based find it difficult to get a clear range of communication in the food depot with minimum number of nodes. Here, an analysis of the proposed topology is provided to establish a relationship between network size and required coverage. The sensing range of sensing devices lies between 25-30 meters (Ammari and Das, 2012). The width of standard depot is approximately 22 meters. Therefore, a sensor deployed on either walls can sense the area in its sectored disc range till the opposite wall. The depot can be divided by sensing zone of each sensor as shown in Figure 5. It can be observed that by placing sensing devices at the center of the sensing disc (shown by dark spots) in the figure, the entire depot can be covered.

Consider a depot of dimension $l \times b \mathrm{~m}^{2}$. Let $r$ and $\theta$ are the sensing range and angle of sensing sector for a sensor respectively. Let $d$ be the length of the chord as shown in Figure 5.

Theorem 1: The number of nodes required by two- 


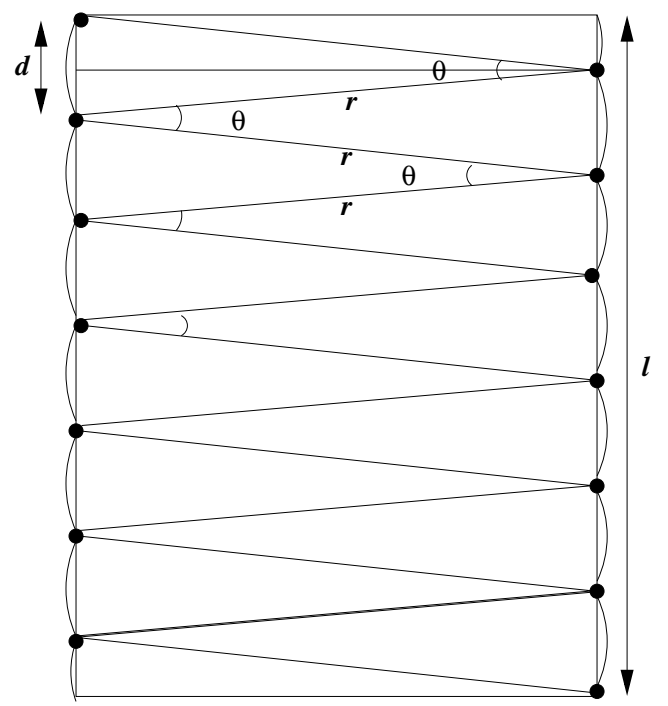

Figure 5: Coverage of depot using sectored sensing disc.

tail chain topology to achieve 1-coverage is:

$$
\left(\frac{l}{r \times \sin \left(\frac{\theta}{2}\right)}+2\right) \text {. }
$$

Proof. From Figure 5, it can be inferred that the total number of sectors required to cover depot of dimension $l \times b$ is the sum of number of sectors facing left wall and number of sectors facing right walls plus 2 additional partial sectors at both ends of the depot.

$$
\text { No. of sectors in depot }=2 \times\left(\frac{l}{d}\right)+2
$$

The value of $d$ can be represented in terms of $r$ and angle $\theta$ as:

$$
d=2 \times r \times \sin \left(\frac{\theta}{2}\right)
$$

Using Equation 3 in 2 we get:

No. of sectors in depot $=2 \times\left(\frac{l}{2 \times r \times \sin \left(\frac{\theta}{2}\right)}\right)+2$

$$
=\left(\frac{l}{r \times \sin \left(\frac{\theta}{2}\right)}\right)+2
$$

From the above Equation, 1-coverage of the depot can be achieved by placing a sensor at each center of the sectors as shown by dark spot in Figure 5. Therefore, number of nodes required to achieve 1-coverage is equal to:

$$
\left(\frac{l}{r \times \sin \left(\frac{\theta}{2}\right)}+2\right)
$$

Theorem 2: Chain density for 1-coverage topology is equal to:

$$
\left(\frac{1}{2 \times r \times \sin \left(\frac{\theta}{2}\right)}+\frac{1}{l}\right) .
$$

Proof. Considering Figure 5, the dimension of the depot is given by $l \times b$. Then, the total length of the chain across the wall is $2 \times l$. Using Equation 5 , the number of nodes required for 1-coverage is given by:

$$
N=\left(\frac{l}{r \times \sin \left(\frac{\theta}{2}\right)}+2\right) .
$$

Therefore, the chain density to achieve 1-coverage is given by:

$$
\begin{aligned}
\text { chain density } & =\frac{N}{2 \times l} \\
& =\frac{\left(\frac{l}{r \times \sin \left(\frac{\theta}{2}\right)}+2\right)}{2 \times l} \\
& =\left(\frac{1}{2 \times r \times \sin \left(\frac{\theta}{2}\right)}+\frac{1}{l}\right) .
\end{aligned}
$$

$\mathrm{k}$-coverage of depot is achieved by increasing chain density for 1-coverage topology by a factor of $k$.

Lemma 1: Chain density for k-coverage topology is equal to:

$$
k \times\left(\frac{1}{2 \times r \times \sin \left(\frac{\theta}{2}\right)}+\frac{1}{l}\right) .
$$

\subsection{Energy Consumption}

This paper follow energy consumption model provided in (Mishra and Samantara, 2016), which is considered for evaluating energy consumption of sensing devices used in proposed topology. Let us assume 2 that the size of data message is $m$ bits. To achieve 1-coverage the number of nodes required by two-tail topology is given by Equation 5. Since the chain

(4) length is taken as $2 \times l$, then the average distance between two successive sensing devices in the chain is given by:

$$
\begin{aligned}
d & =\frac{2 \times l}{N-1} \\
& =\frac{2 \times l}{\left(\frac{l}{r \times \sin \left(\frac{\theta}{2}\right)}+2\right)-1} \\
& =\frac{2 \times l \times r \times \sin \left(\frac{\theta}{2}\right)}{l+r \times \sin \left(\frac{\theta}{2}\right)}
\end{aligned}
$$


The total energy consumed by all devices in the chain in a single round of data transmission is given by:

$$
E=E_{T X}+E_{R X}+E_{A}
$$

where, $E_{T X}, E_{R X}$, and $E_{A}$ are the total energy consumed during data transmission, reception, and aggregation respectively. The energy consumed to transmit a message of $m$ bit over a distance of $d$ is given by:

$$
E_{\text {elec }} \times m+\varepsilon_{\text {amp }} \times m \times d^{2}
$$

where, $E_{\text {elec }}$ and $\varepsilon_{a m p}$ is the energy required to transmit a single bit by the transmitter electronics and the amplifier respectively. Then, $E_{T X}$ is given by:

$$
\begin{aligned}
E_{T X} & =\left(E_{\text {elec }} \times m+\varepsilon_{\text {amp }} \times m \times d^{2}\right) \times N \\
& =\left(E_{\text {elec }} \times m+\varepsilon_{\text {amp }} \times m \times d^{2}\right) \times\left(\frac{l}{r \times \sin \left(\frac{\theta}{2}\right)}+2\right)
\end{aligned}
$$

Similarly, $E_{R X}$ is given by

$$
E_{R X}=\left(E_{\text {elec }} \times m \times\left(\frac{l}{r \times \sin \left(\frac{\theta}{2}\right)}+2\right)\right.
$$

Let $E_{a g}$ be the energy spent for aggregation of $m$-bit data, then

$$
E_{A}=E_{a g} \times\left(\frac{l}{r \times \sin \left(\frac{\theta}{2}\right)}+2\right)
$$

\section{EXPERIMENTAL RESULTS}

The performance of the proposed two-tail chain topology is evaluated by simulation analysis. The parameters of interest are temperature and moisture content (Hu, 2013). Both of these values are varied using physical process module. The proposed topology is compared with mesh and clustered topology using the following metrics: detection accuracy, message delivery ratio, energy consumption, delay, and number of alive nodes in the network. The plot for detection accuracy vs. number of nodes deployed in the network is depicted in Figure 6. It is observed that the detection accuracy of proposed topology raise to hundred percent when number of nodes is greater than or equal to twenty. This accuracy is better than the existing ones. This is because of optimal positioning of sensing devices to sense data uniformly over the food grain storage depot. The comparison of energy consumption over number of rounds is shown in Figure 7. It is observed from the figure that the energy consumption per node of the proposed topology over number of rounds remains almost constant. A marginally lesser consumption is achieved compared

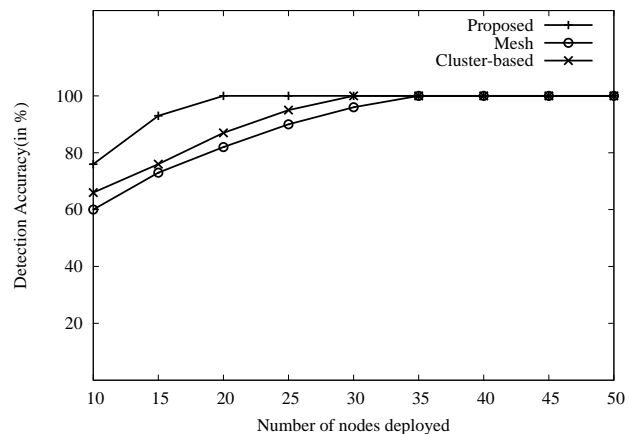

Figure 6: Detection Accuracy vs. Number of nodes deployed in the chain

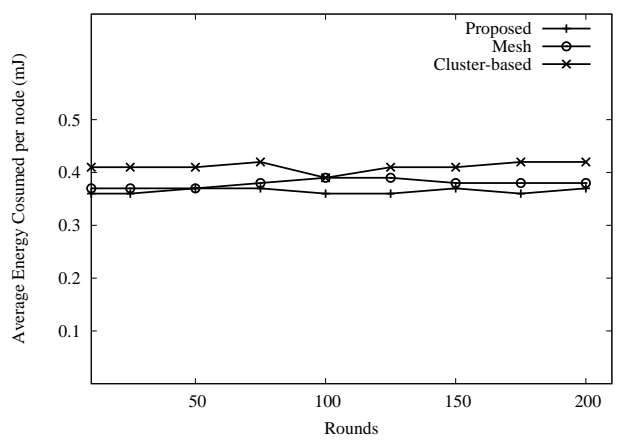

Figure 7: Energy Consumed per node vs. Number of rounds.

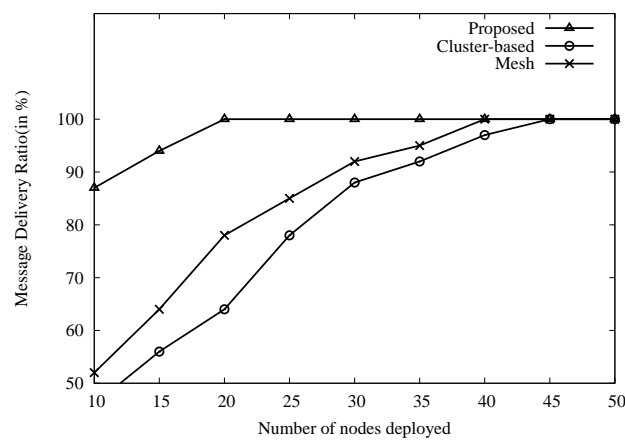

Figure 8: Message delivery ratio with food grain bins inside the depot.

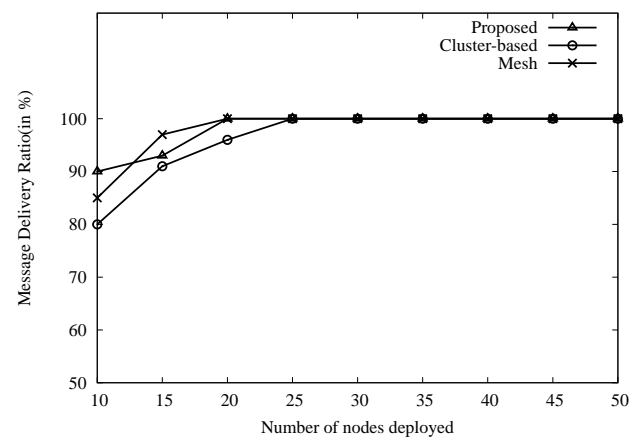

Figure 9: Message delivery ratio without food grain bins inside the depot. 
to mesh and clustered topology due to use of directional antenna for communication.

Figures 8 and 9 show the comparison of message delivery ratio over number of nodes deployed in the network with and without foog grain bins inside the depot, respectively. For the proposed topology, the message delivery ratio is computed with relaying node as the destination node. It is observed that the proposed topology is able to achieve higher message delivery ratio with food grain bins in the depot than mesh and cluster-based topology. This is because, when food grains are placed in the depot, they hinder the communication between the nodes of mesh and clustered topology. However, two-tail chain topology uses routing of messages along the chain and remains unaffected by existence of food grain bins in the depot. Figure 10 compares the number of alive

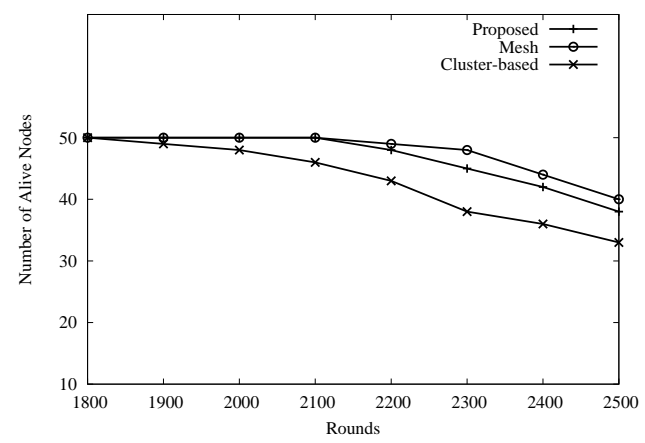

Figure 10: Number of alive nodes vs. Number of rounds.

nodes in the network vs. number of rounds. It is observed that the rate of decreasing in the number of alive nodes in proposed topology is marginally higher than mesh topology and lower than clustered topology. This is because, the proposed topology does not use multi-path routing like mesh topology to balance the remaining energy of the nodes. The higher rate of decrease in the clustered topology is due to significant consumption of energy for inter- $\mathrm{CH}$ communication inside food grain depot. Finally, the com-

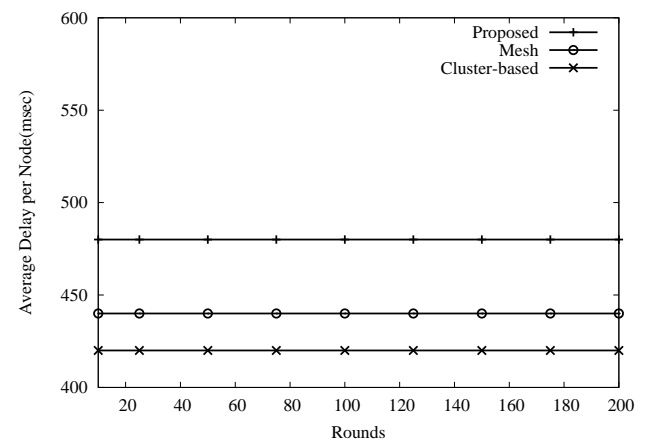

Figure 11: Average delay per node vs. Number of rounds. parison of average delay vs. number of rounds are depicted in the Figure 11. It is observed that the proposed topology incurs higher delay compared to mesh and clustered topology and it remains almost constant over number of rounds. This delay is mostly due to additional waiting time of each node before processing and forwarding the data to the next node.

\section{CONCLUSION}

The proposed two-tail chain topology aims to provide an efficient monitoring process for food grain depot. The proposed topology is designed based on the standard infrastructure of food grain depots and other environmental constraints. Due to open space constraint the proposed topology use a chain structure to deploy sensing devices on the inner walls of the depot. To achieve efficient sensing and communication in this environment, the sensing devices are equipped with directional communication antenna instead of omni-directional antenna. This helps to achieve a clear line of sight for sensing and communicating data inside the food grain depot. The performance of the proposed topology is compared with that for mesh and clustered topology. The results of detection accuracy, message delivery ratio, and energy consumption infers the suitability of the proposed topology over existing ones for monitoring food grains.

Further, we are aiming to deploy proposed two-tail chain topology in real-time testbed for better evaluation our topology.

\section{ACKNOWLEDGEMENTS}

An initial short version of this work has been published in the proceedings of 5th International Conference on Advanced Computing, Networking, and Informatics (ICACNI), June 2017 (Tripathy et al., 2017).

\section{REFERENCES}

Alsemairi, S. and Younis, M. (2016). Forming a ClusterMesh Topology to Boost Base-Station Anonymity in Wireless Sensor Networks. In Proceedings of IEEE Wireless Communications and Networking Conference, WCNC 2016, pages $01-06$.

Ammari, H. M. and Das, S. K. (2012). Centralized and Clustered k-Coverage Protocols for Wireless Sensor Networks. IEEE Transactions on Computers, 61(1).

Aziz, A. A., Sekercioglu, Y. A., Fitzpatrick, P., and Ivanovich, M. (2013). A Survey on Distributed Topology Control Techniques for Extending the Lifetime of 
Battery Powered Wireless Sensor Networks. IEEE Communications Surveys \& Tutorials, 15(1):121 144.

Carlos-Mancilla, M., Lpez-Mellado, E., and Siller, M. (2016). Wireless Sensor Networks Formation: Approaches and Techniques. Journal of Sensors, 2016(2016): 1 - 18.

Ceclio, J. and Furtado, P. (2014). Wireless Sensors in Heterogeneous Networked Systems, chapter Wireless Sensor Networks: Concepts and Components, pages 5 25. Springer.

Deshpande, N., Shaligram, A. D., Botre, B. A., Bindal, S., and Sadistap, S. . (2010). Embedded E-nose Application to Sense the Food Grain Storage Condition. In Proceedings of International Conference of Computational Intelligence and Communication Networks, pages $608-611$.

Fan, G. J. and Jin, S. (2010). Coverage Problem in Wireless Sensor Network: A Survey. Journal of Networks, 5(9): 1033 - 1040.

Hadjidj, A., Souil, M., Bouabdallah, A., Challal, Y., and Owen, H. (2013). Wireless sensor networks for rehabilitation applications: Challenges and opportunities. Journal of Network and Computer Applications, 36(1):01 - 15 .

Hellevang, K. J. (2010). Grain drying. NDSU Extension Service, Fargo, ND (1994).

Hong, Z., Wang, R., and Li, X. (2016). A Clusteringtree Topology Control Based on the Energy Forecast for Heterogeneous Wireless Sensor Networks. 68 IEEE/CAA JOURNAL OF AUTOMATICA SINICA, $3(1): 68-77$.

Hu, F. (2013). Tele-Healthcare Computing and Engineering: Principles and Design, chapter Hardware: Sensor Mote Architecture and Design. SCIENCE PUBLISHERS, CRC Press.

Manay, N. and M.Shadaksharaswamy (2008). Foods Facts and Principles. New Age International, 3rd edition.

Mishra, A. K. and Samantara, M. K. (2016). A dynamic energy-efficient chain formation scheme for PEGASIS in wireless sensor networks. In Proceedings of 2nd International Conference on Computational Intelligence \& Networks, CINE 2016, pages $41-46$.

Puthal, D., Malik, N., Mohanty, S. P., Kougianos, E., and Yang, C. (2018). The blockchain as a decentralized security framework. IEEE Consumer Electronics Magazine, 7(2):18-21.

Roy, S. S., Puthal, D., Sharma, S., Mohanty, S. P., and Zomaya, A. Y. (2018). Building a sustainable internet of things: Energy-efficient routing using low-power sensors will meet the need. IEEE Consumer Electronics Magazine, 7(2):42-49.

Sahoo, B., Rath, S., and Puthal, D. (2012). Energy efficient protocols for wireless sensor networks: A survey and approach. International Journal of Computer Applications, 44(18):43-48.

Sawant, A., Patil, S. C., Kalse, S. B., and Thakor, N. J. (2012). Effect of temperature, relative humidity and moisture content on germination percentage of wheat stored in different storage structures. AgricEngInt: CIGR Journal, 14(2):01 - 14.

Sharma, S., Puthal, D., Jena, S. K., Zomaya, A. Y., and Ranjan, R. (2017a). Rendezvous based routing protocol for wireless sensor networks with mobile sink. The Journal of Supercomputing, 73(3):1168-1188.

Sharma, S., Puthal, D., Tazeen, S., Prasad, M., and Zomaya, A. Y. (2017b). Msgr: A mode-switched grid-based sustainable routing protocol for wireless sensor networks. IEEE Access, 5:19864-19875.

Tanwar, S., Kumar, N., and Rodrigues, J. J. (2015). A systematic review on heterogeneous routing protocols for wireless sensor network. Journal of Network and Computer Applications, 53(0):39 - 56.

Tripathy, A. K., kumar Mishra, A., Kumar, A., and Das, T. (2017). A Two-Tailed Chain Topology in Wireless Sensor Networks for Efficient Monitoring of Food Grain Storage. In Proceedings of 5th International Conference on Advanced Computing, Networking, and Informatics (ICACNI).

Yang, C., Puthal, D., Mohanty, S. P., and Kougianos, E. (2017). Big-sensing-data curation for the cloud is coming: A promise of scalable cloud-data-center mitigation for next-generation iot and wireless sensor networks. IEEE Consumer Electronics Magazine, $6(4): 48-56$

Yu, Z., Teng, J., Bai, X., Xuan, D., and Jia, W. (2011). Connected coverage in wireless networks with directional antennas. In Proceedings of IEEE INFOCOM, pages 2264-2272.

Zin, S. M., Anuar, N. B., Kiah, M. L. M., and Pathan, A.-S. K. (2014). Routing protocol design for secure WSN: Review and open research issues. Journal of Network and Computer Applications, 41(0):517 530. 